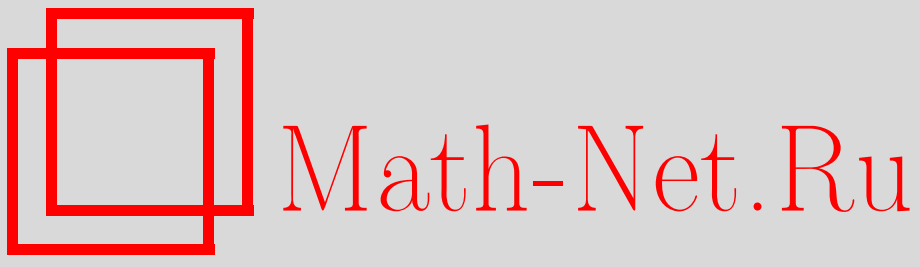

I. Fazekas, O. Klesov, A general approach to the strong laws of large numbers, Теория вероятн. и ее примен., 2000, том 45, выпуск 3, 568-583

DOI: https://doi.org/10.4213/tvp486

Использование Общероссийского математического портала Math-Net.Ru подразумевает, что вы прочитали и согласны с пользовательским соглашением http://www.mathnet.ru/rus/agreement

Параметры загрузки:

IP: 3.80 .253 .173

26 апреля 2023 г., 13:53:34

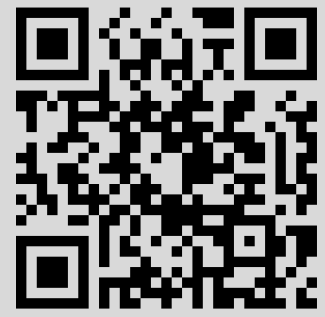


(c) $2000 \mathrm{r}$.

FAZEKAS I. ${ }^{*}$ KLESOV O.**

\title{
A GENERAL APPROACH TO THE STRONG LAWS OF LARGE NUMBERS ${ }^{1)}$
}

\begin{abstract}
Рассматривается общий метод получения усиленного закона больших чисел для последовательностей случайных величин. Даются некоторые приложения для случая зависимых слагаемых.
\end{abstract}

Ключевые слова и фразы: усиленный закон больших чисел, максимальное неравенство Гаека Реньи, $\rho$-перемешивание, логарифмически взвешенные суммы.

0. Introduction. The strong law of large numbers (SLLN) asserts that a sequence of cumulative sums of random variables becomes «nonrandom» by normalizing it by an appropriate sequence of nonrandom numbers and approaching the limit. Many results of this type were obtained for both independent and dependent summands forming cumulative sums.

There are two basic approaches to prove the strong law of large numbers. The first of them is to prove the result desired for a subsequence and then reduce the problem for the whole sequence to the one for the subsequence. In so doing, a maximal inequality for cumulative sums is usually needed for the second step. Note that maximal inequalities are a well-developed branch of probability theory and many inequalities are known for different classes of random variables.

The second approach is to use directly a maximal inequality for normed sums. Inequalities of this kind are said to be of Hájek-Rényi type. The notion refers to the paper by Hájek and Rényi [5] devoted to independent summands. Inequalities of this type are not easy to obtain and the first approach prevails. However, after a Hájek-Rényi inequality is obtained the proof of the strong laws of large numbers becomes an obvious problem. Some of Hájek-Rényi inequalities were announced in [8].

\footnotetext{
${ }^{*}$ Institute of Mathematics and Informatics, Kossuth Lajos University, P. O. Box 12, H-4010 Debrecen, Hungary; e-mail: fazekasi@math.klte.hu

${ }^{* *}$ National Technical University of Ukraine (KPI), Department of Mathematics \#1, pr. Peremogy 37, Kiev 252056, Ukraine; e-mail: oleg@tbimc.freenet.kiev.ua

1) Supported by the Hungarian Ministry of Culture and Education under Grant № FKFP 0429-1997; the research was partially realized while the second author was visiting Institute of Mathematics and Informatics, Kossuth University, Debrecen, Hungary.
} 
In this paper, our program is to show that a Hájek-Rényi type inequality is, in fact, a consequence of an appropriate maximal inequality for cumulative sums and that the latter automatically implies the strong law of large numbers. The most important is that we made no restriction on the dependence structure of random variables. To fulfill this program we prove two basic theorems. Several examples of applications are given in separate sections. We do not consider orthogonal and stationary dependence structure because results in these cases are well-known, however these are two of possible applications of our general approach. Also we do not discuss in detail the case of independent summands, however, they appear in several parts of the paper as an example of the origin of the theory.

The paper is organized as follows. In the first two sections following this Introduction, we give the Hájek-Rényi inequality and prove the strong law of large numbers for general summands. We stress that no assumption on dependence between summands is made and the only condition posed is that a certain moment of individual summands exists. The well-known Kolmogorov theorem on the strong law of large numbers for nonidentically distributed independent random variables is a simple corollary of our general result.

In the six subsequent sections we consider several applications of the above results. In Section 3, we prove an extension and sharpening of the Brunk-Prokhorov theorem. The extension is that this theorem is proved for martingales and sharpening is that we give the strong law of large numbers for a general normalizing sequence. In Section 4, we give an estimate of the order of growth of cumulative sums in a manner introduced by Petrov [13] for independent summands. The most interesting fact is that this result is obtained without preassumed dependence structure. In Section 5, we consider $\rho$-mixing sequences. The results presented there differ from those known in the literature because they cover the cases $\sum \rho\left(2^{i}\right)=\infty$ and $\sup _{n \geqslant 1} \mathbf{E} X_{n}^{2}=\infty$. As a corollary of the strong law of large numbers we obtain an estimate of the order of growth of cumulative sums of a $\rho$-mixing sequence. In Section 6, results for mixingales are established. We briefly discuss in Section 7 applications for sequences whose moment functions have a superadditive structure. Section 8 is devoted to a special normalizing sequence $\left\{b_{n}\right\}$, namely $b_{n}=\ln n$. Results given in Section 8 may lead to the so called strong central limit theorems. The approach presented seems to be suitable even in more general situations.

We use the following notation. $X_{1}, X_{2}, \ldots$ denote a sequence of random variables defined on a fixed probability space. The partial sums of the random variables are $S_{n}=\sum_{i=1}^{n} X_{i}$ for $n \geqslant 1$ and $S_{0}=0$. A sequence $\left\{b_{n}\right\}$ is called nondecreasing if $b_{i} \leqslant b_{i+1}$ for $i \geqslant 1$.

Before entering the precise mathematical statements, the second author expresses his sincere gratitude for the hospitality provided to him during his visit to Kossuth University, Debrecen. 


\section{Häjek-Rényi type maximal inequality.}

Theorem 1.1. Let $\beta_{1}, \ldots, \beta_{n}$ be a nondecreasing sequence of positive numbers. Let $\alpha_{1}, \ldots, \alpha_{n}$ be non-negative numbers. Let $r$ be a fixed positive number. Assume that for each $m$ with $1 \leqslant m \leqslant n$

$$
\mathbf{E}\left[\max _{1 \leqslant l \leqslant m}\left|S_{l}\right|\right]^{r} \leqslant \sum_{l=1}^{m} \alpha_{l}
$$

Then

$$
\mathbf{E}\left[\max _{1 \leqslant l \leqslant n}\left|\frac{S_{l}}{\beta_{l}}\right|\right]^{r} \leqslant 4 \sum_{l=1}^{n} \frac{\alpha_{l}}{\beta_{l}^{r}} .
$$

$\mathrm{P}$ r o of. We can suppose that $\beta_{1}=1$. Let $c=2^{1 / r}$. Consider the following sets $A_{i}=\left\{k: c^{i} \leqslant \beta_{k}<c^{i+1}\right\}, i=0,1,2, \ldots$ Denote by $i(n)$ the index of the last non-empty $A_{i}$. Let $k(i)=\max \left\{k: k \in A_{i}\right\}, i=0,1,2, \ldots$, if $A_{i}$ is non-empty, while $k(i)=k(i-1)$ if $A_{i}$ is empty and let $k(-1)=0$. Let

$$
\delta_{l}=\sum_{j=k(l-1)+1}^{k(l)} \alpha_{j}, \quad l=0,1,2, \ldots,
$$

where $\delta_{l}$ is considered to be zero if $A_{l}$ is empty. We have

$$
\begin{aligned}
\mathbf{E}\left[\max _{1 \leqslant l \leqslant n}\left|\frac{S_{l}}{\beta_{l}}\right|\right]^{r} & \leqslant \sum_{i=0}^{i(n)} \mathbf{E}\left[\max _{l \in A_{i}}\left|\frac{S_{l}}{\beta_{l}}\right|\right]^{r} \leqslant \sum_{i=0}^{i(n)} c^{-i r} \mathbf{E}\left[\max _{l \in A_{i}}\left|S_{l}\right|\right]^{r} \\
& \leqslant \sum_{i=0}^{i(n)} c^{-i r} \mathbf{E}\left[\max _{k \leqslant k(i)}\left|S_{k}\right|\right]^{r} \leqslant \sum_{i=0}^{i(n)} c^{-i r} \sum_{k=1}^{k(i)} \alpha_{k} \\
& =\sum_{i=0}^{i(n)} c^{-i r} \sum_{l=0}^{i} \delta_{l}=\sum_{l=0}^{i(n)} \delta_{l} \sum_{i=l}^{i(n)} c^{-i r} \leqslant \sum_{l=0}^{i(n)} \delta_{l} \sum_{i=l}^{\infty} c^{-i r} \\
& \leqslant \frac{1}{1-c^{-r}} \sum_{l=0}^{i(n)} \delta_{l} c^{-l r}=\frac{1}{1-c^{-r}} \sum_{l=0}^{i(n)} c^{-l r} \sum_{k=k(l-1)+1}^{k(l)} \alpha_{k} \\
& \leqslant \frac{c^{r}}{1-c^{-r}} \sum_{l=0}^{i(n)} \sum_{k=k(l-1)+1}^{k(l)} \frac{\alpha_{k}}{\beta_{k}^{r}}=4 \sum_{k=1}^{n} \frac{\alpha_{k}}{\beta_{k}^{r}} .
\end{aligned}
$$

The theorem is proved.

\section{Strong law of large numbers.}

Theorem 2.1. Let $b_{1}, b_{2}, \ldots$ be a nondecreasing unbounded sequence of positive numbers. Let $\alpha_{1}, \alpha_{2}, \ldots$ be non-negative numbers. Let $r$ be a fixed positive number. Assume that for each $n \geqslant 1$

$$
\mathbf{E}\left[\max _{1 \leqslant l \leqslant n}\left|S_{l}\right|\right]^{\tau} \leqslant \sum_{l=1}^{n} \alpha_{l}
$$


If $\sum_{l=1}^{\infty}\left(\alpha_{l} / b_{l}^{r}\right)<\infty$ then

$$
\lim _{n \rightarrow \infty} \frac{S_{n}}{b_{n}}=0 \quad \text { a.s. }
$$

$\mathrm{P}$ r o o f. First we assume that there exists an integer $m$ such that $\alpha_{n}=0, n \geqslant m$. Then $\mathbf{E}\left[\sup _{n \geqslant 1}\left|S_{n}\right|\right]^{r}<\infty$ via (2.1) and this easily gives (2.2). Therefore we assume in what follows that $\alpha_{n}>0$ for infinite number of indices $n$. Set

$$
v_{n}=\sum_{k=n}^{\infty} \frac{\alpha_{k}}{b_{k}^{\tau}}, \quad \beta_{n}=\max _{1 \leqslant k \leqslant n} b_{k} v_{k}^{1 / 2 r} .
$$

It is easy to see that $0<v_{n}<\infty$ for all $n \geqslant 1$. Using Dini's theorem, see $[4, \S 375.5]$, we prove that $\sum \alpha_{n} / b_{n}^{r} v_{n}^{1 / 2}<\infty$. So the sequence $\left\{\beta_{n}, n \geqslant 1\right\}$ is such that (a) $\beta_{k} \leqslant \beta_{k+1}, k=1,2, \ldots$, (b) $\sum_{k=1}^{\infty}\left(\alpha_{k} / \beta_{k}^{r}\right)<\infty$, (c) $\lim _{k \rightarrow \infty}\left(\beta_{k} / b_{k}\right)=0$. Then Theorem 1.1 implies that (1.2) is satisfied. Therefore

$$
\mathbf{E}\left[\sup _{l \geqslant 1}\left|\frac{S_{l}}{\beta_{l}}\right|\right]^{r} \leqslant 4 \sum_{l=1}^{\infty} \frac{\alpha_{l}}{\beta_{l}^{r}}<\infty .
$$

This implies $\sup _{l \geqslant 1}\left|S_{l} / \beta_{l}\right|<\infty$ a.s. Finally,

$$
0 \leqslant\left|\frac{S_{l}}{b_{l}}\right|=\left|\frac{S_{l}}{\beta_{l}}\right| \frac{\beta_{l}}{b_{l}} \leqslant\left\{\sup _{l \geqslant 1}\left|\frac{S_{l}}{\beta_{l}}\right|\right\} \frac{\beta_{l}}{b_{l}} \longrightarrow 0 \quad \text { a.s. as } l \rightarrow \infty .
$$

The theorem is proved.

Corollary 2.1. Let $b_{1}, b_{2}, \ldots$ be a nondecreasing unbounded sequence of positive numbers. Let $\alpha_{1}, \alpha_{2}, \ldots$ be non-negative numbers, $\Lambda_{k}=\alpha_{1}+\cdots+\alpha_{k}$ for $k \geqslant 1$. Let $r$ be a fixed positive number. Assume that for each $n \geqslant 1$ (2.1) is satisfied. If

$$
\begin{array}{r}
\sum_{l=1}^{\infty} \Lambda_{l}\left(\frac{1}{b_{l}^{r}}-\frac{1}{b_{l+1}^{r}}\right)<\infty \\
\frac{\Lambda_{n}}{b_{n}^{\tau}} \quad \text { is bounded }
\end{array}
$$

then (2.2) is fulfilled.

$\mathrm{R}$ e $\mathrm{m}$ a r $\mathrm{k}$ 2.1. In some cases condition (2.4) is a consequence of (2.3). Let, for instance, $b_{n}^{\tau}=n^{\delta}, \delta>0$. Then (2.3) is equivalent to $\sum \Lambda_{n} n^{-(1+\delta)}<\infty$ and therefore

$$
\infty>\sum_{n=1}^{\infty} \frac{\Lambda_{n}}{n^{1+\delta}}=\sum_{m=0}^{\infty} \sum_{2^{m} \leqslant n<2^{m+1}} \frac{\Lambda_{n}}{n^{1+\delta}} \geqslant \frac{1}{2} \sum_{m=0}^{\infty} \frac{\Lambda_{2^{m}}}{2^{(m+1) \delta}}
$$


since $\Lambda_{n}$ is nondecreasing. Hence $\Lambda_{2^{m}} /\left(2^{m}\right)^{\delta} \rightarrow 0$. The same is true for the whole sequence:

$$
0 \leqslant \max _{2^{m} \leqslant n<2^{m+1}} \frac{\Lambda_{n}}{n^{\delta}} \leqslant 2^{\delta} \frac{\Lambda_{2^{m+1}}}{2^{(m+1) \delta}} \rightarrow 0 .
$$

3. Brunk-Prokhorov theorem for martingales. Let $q \geqslant 1$. For independent random variables $\left\{X_{n}, n \geqslant 1\right\}$ Brunk [2] for integer $q$ and Prokhorov [14] for the general case proved the following result: if $\mathbf{E} X_{n}=0$, $\mathbf{E}\left|X_{n}\right|^{2 q}<\infty, n \geqslant 1$, and $\sum_{n=1}^{\infty} \mathbf{E}\left|X_{n}\right|^{2 q} / n^{q+1}<\infty$ then

$$
\lim _{n \rightarrow \infty} \frac{S_{n}}{n}=0 \quad \text { a.s. }
$$

Using the general approach described in previous sections, it is easy to get the same result for martingales and for more general norming sequences. Namely, we prove

Theorem 3.1. Let $\left\{X_{n}, n \geqslant 1\right\}$ be a martingale-difference with respect to the filtration $\left\{\mathscr{F}_{n}, n \geqslant 1\right\}$, where the $\sigma$-field $\mathscr{F}_{n}$ is generated by the random variables $X_{1}, \ldots, X_{n}$. Assume $q>\frac{1}{2}$ and let $\left\{b_{n}, n \geqslant 1\right\}$ be a nondecreasing unbounded sequence. If

$$
\sum_{n=1}^{\infty} \frac{\mathbf{E}\left|S_{n}\right|^{2 q}-\mathbf{E}\left|S_{n-1}\right|^{2 q}}{b_{n}^{2 q}}<\infty
$$

then

$$
\lim _{n \rightarrow \infty} \frac{S_{n}}{b_{n}}=0 \quad \text { a.s. }
$$

The proof of Theorem 3.1 easily follows from Theorem 2.1 by noting that $\mathbf{E}\left|S_{n}\right|^{2 q}$ increases because of the submartingale property, moreover

$$
\mathbf{E}\left[\max _{k \leqslant n}\left|S_{k}\right|\right]^{2 q} \leqslant\left(\frac{2 q}{2 q-1}\right)^{2 q} \mathbf{E}\left|S_{n}\right|^{2 q}
$$

according to a Doob inequality.

A Brunk-Prokhorov type statement may be given as follows.

Corollary 3.1. Let $\left\{X_{n}, n \geqslant 1\right\}$ be a martingale-difference with respect to the filtration $\left\{\mathscr{F}_{n}, n \geqslant 1\right\}$, where the $\sigma$-field $\mathscr{F}_{n}$ is generated by the random variables $X_{1}, \ldots, X_{n}$. Let $\left\{b_{n}, n \geqslant 1\right\}$ be a nondecreasing unbounded sequence. Assume either $q=1$ or $q>1$ and $n^{-\delta} b_{n}$ is nondecreasing for some $\delta>(q-1) /(2 q)$. If

$$
\sum_{n=1}^{\infty} \frac{\mathbf{E}\left|X_{n}\right|^{2 q}}{b_{n}^{2 q}} n^{q-1}<\infty
$$

then (3.3) holds. 
$\mathrm{R}$ e $\mathrm{m}$ a $\mathrm{r}$ k 3.1. Corollary 3.1 is well-known for $q=1$, see, for instance, $\left[15\right.$, Theorem 3.3.1]. Moreover, the condition that $n^{-\delta} b_{n}$ is nondecreasing may be omitted in this case.

P r o of of $\mathrm{C}$ or o $11 \mathrm{ar}$ y 3.1. We use a Burkholder [3] inequality: there exists a constant $A_{q}$ such that

$$
\mathbf{E}\left|S_{n}\right|^{2 q} \leqslant A_{q} \mathbf{E}\left[\sum_{k=1}^{n} X_{k}^{2}\right]^{q}
$$

Now, we apply the Hölder inequality if $q>1: \mathbf{E}\left|S_{n}\right|^{2 q} \leqslant$ $A_{q} n^{q-1} \sum_{k=1}^{n} \mathbf{E}\left|X_{k}\right|^{2 q}$, which is valid for $q \geqslant 1$. By (3.4), we obtain

$$
\mathbf{E}\left[\max _{k \leqslant n}\left|S_{k}\right|\right]^{2 q} \leqslant \sum_{k=1}^{n} \alpha_{k}
$$

where $\alpha_{1}=c \mathbf{E}\left|X_{1}\right|^{2 q}$ and

$$
\alpha_{k}=c\left(k^{q-1} \sum_{j=1}^{k} \mathbf{E}\left|X_{j}\right|^{2 q}-(k-1)^{q-1} \sum_{j=1}^{k-1} \mathbf{E}\left|X_{j}\right|^{2 q}\right), \quad k>1
$$

(here $c$ is an appropriate constant not depending on $k$ ). Then we have

$$
\alpha_{n} \leqslant \text { const }\left[n^{q-1} \mathbf{E}\left|X_{n}\right|^{2 q}+n^{q-2} \sum_{k=1}^{n-1} \mathbf{E}\left|X_{k}\right|^{2 q}\right] \text {, }
$$

where the second summand should be omitted in the case $q=1$. So, we have

$$
\sum_{n=1}^{\infty} \frac{\alpha_{n}}{b_{n}^{2 q}} \leqslant \text { const }\left[\sum_{n=1}^{\infty} \frac{\mathbf{E}\left|X_{n}\right|^{2 q}}{b_{n}^{2 q}} n^{q-1}+\sum_{n=1}^{\infty} \frac{n^{q-2}}{b_{n}^{2 q}} \sum_{k=1}^{n-1} \mathbf{E}\left|X_{k}\right|^{2 q}\right],
$$

where the second series on the right-hand side should be omitted in the case $q=1$. This proves the result in the case $q=1$. Otherwise, if $q>1$, we have

$$
\sum_{n=1}^{\infty} \frac{n^{q-2}}{b_{n}^{2 q}} \sum_{k=1}^{n-1} \mathbf{E}\left|X_{k}\right|^{2 q}=\sum_{k=1}^{\infty} \mathbf{E}\left|X_{k}\right|^{2 q} \sum_{n=k+1}^{\infty} \frac{n^{q-2}}{b_{n}^{2 q}} \leqslant \text { const } \sum_{k=1}^{\infty} \frac{\mathbf{E}\left|X_{k}\right|^{2 q}}{b_{k}^{2 q}} k^{q-1}
$$

because $n^{-\delta} b_{n}$ increases if $\delta>(q-1) /(2 q)$. Theorem 2.1 gives the result.

4. An estimate of the order of growth of sums. V. V. Petrov has developed an approach allowing one to estimate the order of growth of partial sums in terms of their «natural» characteristics (see [13]). In many cases these characteristics are presented by sums of moments of individual random summands. This is the case, for instance, for independent, orthogonal, and 
stationary sequences. Below we give a generalization of such results for the case of general random variables. The price we pay for this generalization is that the «natural» characteristics become moments of $\max _{k \leqslant n}\left|S_{k}\right|$. We note, however, that no assumption is made on the dependence structure but, using appropriate (well-known) maximal inequalities, results may be expressed in simpler terms for many particular cases of random sequences.

Following $\left[13\right.$, Ch. IX, Section 3], we define the class $\Psi_{c}$ of functions $\psi(x)$ such that

(i) there exists an integer number $n_{0} \geqslant 0$ (depending on the function $\psi$ ) such that $\psi(x)$ is nondecreasing in $x \in\left(n_{0}, \infty\right)$,

(ii) $\sum_{n=n_{0}}^{\infty} 1 /(n \psi(n))<\infty$.

Theorem 4.1. Let $p>0$. Let $\left\{X_{n}, n \geqslant 1\right\}$ be a sequence of random variables such that $\mathbf{E}\left|X_{n}\right|^{p}<\infty, n \geqslant 1$. Let $A_{n}=\mathbf{E}\left[\max _{k \leqslant n}\left|S_{k}\right|\right]^{p}$ and suppose that $\lim _{n \rightarrow \infty} A_{n}=\infty$. Then for any function $\psi \in \Psi_{c}$

$$
\lim _{n \rightarrow \infty} \frac{S_{n}}{\left(A_{n} \psi\left(A_{n}\right)\right)^{1 / p}}=0 \quad \text { a.s. }
$$

P r o of. Set $\alpha_{1}=A_{1}$ and $\alpha_{k}=A_{k}-A_{k-1}, k>1$. Then obviously $\mathbf{E}\left[\max _{k \leqslant n}\left|S_{k}\right|\right]^{p} \leqslant \sum_{k=1}^{n} \alpha_{k}$ and we may apply Theorem 2.1 with $b_{k}=\left(A_{k} \psi\left(A_{k}\right)\right)^{1 / p}$. The series $\sum \alpha_{k} / b_{k}^{p}$ is convergent, since

$$
\sum_{k=\max \left\{2, n_{0}\right\}}^{\infty} \frac{\alpha_{k}}{b_{k}^{p}}=\sum_{k=\max \left\{2, n_{0}\right\}}^{\infty} \frac{A_{k}-A_{k-1}}{A_{k} \psi\left(A_{k}\right)}
$$

and the series on the right-hand side converges due to Lemma 15 of Chapter IX in [13]. Therefore the proof is completed.

We mention some particular cases of Theorem 4.1. For any $\varepsilon>0$

$$
\begin{gathered}
\lim _{n \rightarrow \infty} \frac{S_{n}}{\left(A_{n}^{1+\varepsilon}\right)^{1 / p}}=0 \quad \text { a.s., } \\
\lim _{n \rightarrow \infty} \frac{S_{n}}{\left(A_{n}\left(\ln A_{n}\right)^{1+\varepsilon}\right)^{1 / p}}=0 \quad \text { a.s., } \\
\lim _{n \rightarrow \infty} \frac{S_{n}}{\left(A_{n} \ln A_{n}\left(\ln \ln A_{n}\right)^{1+\varepsilon}\right)^{1 / p}}=0 \quad \text { a.s., and so on. }
\end{gathered}
$$

5. $\rho$-mixing sequences. Let $\left\{X_{n}, n \geqslant 1\right\}$ be a sequence of random variables. Let $\rho(n)$ be its Kolmogorov-Rozanov mixing coefficient, that is,

$$
\rho(n)=\sup _{\substack{k \geqslant 1 \\ X \in L_{1}^{k}, Y \in L_{k+n}^{\infty}}} \frac{\operatorname{cov}(X, Y)}{\sqrt{\mathbf{D} X \cdot \mathbf{D} Y}},
$$

where $L_{i}^{j}$ is the space of square integrable random variables that are measurable with respect to the $\sigma$-field $\mathscr{F}_{i}^{j}$ generated by $X_{i}, \ldots, X_{j}$. In the case 
$j=\infty$ the $\sigma$-field $\mathscr{F}_{i}^{\infty}$ is supposed to be generated by the random variables $X_{i}, X_{i+1}, \ldots$ A sequence of random variables is said to be $\rho$-mixing if its Kolmogorov-Rozanov mixing coefficient $\rho(n)$ tends to zero, as $n \rightarrow \infty$.

Using $\rho(n)$, Shao [16] proved the following result: if $q \geqslant 2$ and $\mathbf{E} X_{n}=0$, $\mathbf{E}\left|X_{n}\right|^{q}<\infty, n \geqslant 1$, then there exists a constant $\theta$ depending only on $q$ and $\{\rho(n)\}$ such that

$$
\begin{aligned}
\mathbf{E}\left[\max _{k \leqslant n}\left|S_{k}\right|\right]^{q} \leqslant \theta[ & n^{q / 2} \exp \left\{\theta \sum_{i=0}^{[\ln n]} \rho\left(2^{i}\right)\right\} \max _{j \leqslant n}\left(\mathbf{E} X_{k}^{2}\right)^{q / 2} \\
& \left.+n \exp \left\{\theta \sum_{i=0}^{[\ln n]} \rho^{2 / q}\left(2^{i}\right)\right\} \max _{j \leqslant n} \mathbf{E}\left|X_{j}\right|^{q}\right] .
\end{aligned}
$$

The simplest case of this inequality is presented by $q=2$. Indeed, for $q=2$ we have

$$
\mathbf{E}\left[\max _{k \leqslant n}\left|S_{k}\right|\right]^{2} \leqslant 2 \theta n \exp \left\{\theta \sum_{i=0}^{[\ln n]} \rho\left(2^{i}\right)\right\} \max _{j \leqslant n} \mathbf{E} X_{j}^{2} .
$$

Among other applications of these inequalities Shao [16] obtains a SLLN: if $1 \leqslant p<2$ then

$$
\lim _{n \rightarrow \infty} \frac{S_{n}}{n^{1 / p}}=0 \quad \text { a.s. }
$$

provided that

(i) $\sum \rho\left(2^{i}\right)<\infty$,

(ii) $X_{n}$ 's are identically distributed,

(iii) $\mathbf{E} X_{n}=0, n \geqslant 1$,

(iv) $\mathbf{E}\left|X_{n}\right|^{p}<\infty, n \geqslant 1$

We give an extension of this result avoiding condition (i). Furthermore, we get SLLN for nonidentically distributed random variables. In particular, we obtain a «real» rate of growth of $S_{n}$. In so doing, we follow our general approach and inequalities (5.1)-(5.2).

Theorem 5.1. Let the sequence $\left\{X_{n}, n \geqslant 1\right\}$ have KolmogorovRozanov coefficient $\rho(n)$. Assume that the constant $\theta$ is defined in (5.2) and $1 \leqslant p<2$. Set $\ln ^{+} t=\ln \max \{e, t\}$ and

$$
\varphi(t)=\sum_{i=0}^{\left[\ln ^{+} t\right]} \rho\left(2^{i}\right)
$$

Assume that $\exp \{\varphi(t)\}$ is a regularly varying function with an index to be small enough, that is, $\exp \{\varphi(t)\}=t^{\varepsilon} L(t)$, where $\varepsilon<(2-p) / p \theta$ and $L(t)$ is a slowly varying function. If

(a) $X_{n}$ 's are identically distributed, 
(b) $\mathbf{E} X_{n}=0, n \geqslant 1$,

(c) $\mathbf{E}\left|X_{n}\right|^{p} \exp \left\{\theta \varphi\left(\left|X_{n}\right|^{p}\right)\right\}<\infty, n \geqslant 1$, then (5.3) holds.

$\mathrm{R}$ e $\mathrm{m}$ a $\mathrm{r} \mathrm{k}$ 5.1. Note that Theorem 5.1 coincides with the above mentioned Shao SLLN if (i) is satisfied. The case $\rho\left(2^{i}\right) \asymp 1 / i$ presents $^{2)}$ an example, where $\sum \rho\left(2^{i}\right)$ diverges but Theorem 5.1 still works. Indeed, $\varphi(t) \asymp \ln \ln t$ in this case and it is easy to see that the proof of Theorem 5.1 remains true if the condition $\exp \{\varphi(t)\}=t^{\varepsilon} L(t)$ is replaced with the condition $\exp \{\varphi(t)\} \asymp t^{\varepsilon} L(t)$ that is satisfied. So, condition (c) becomes $\mathbf{E}\left|X_{n}\right|^{p}\left(\ln ^{+}\left|X_{n}\right|\right)^{\theta L}<\infty, n \geqslant 1$, where $L$ is the upper constant in the relation $\rho\left(2^{i}\right) \asymp 1 / i$. If a precise value of $L$ is not available, we may use, for instance, more restrictive condition $\mathbf{E}\left|X_{n}\right|^{p}\left(\ln ^{+}\left|X_{n}\right|\right)^{\alpha}<\infty, n \geqslant 1$, for all $\alpha>0$ that involves neither $\theta$ nor $L$.

There are other cases, where Theorem 5.1 gives new results compared to Shao's theorem. One more example is given by $\rho\left(2^{i}\right) \asymp$ $(i \ln i)^{-1}$. The strong law of large numbers (5.3) follows from the condition $\mathbf{E}\left|X_{n}\right|^{p}\left(\ln ^{+} \ln ^{+}\left|X_{n}\right|\right)^{\alpha}<\infty, n \geqslant 1$, for all $\alpha>0$. Note that $\rho\left(2^{i}\right) \nrightarrow 0$ in the second case.

P r o of of $\mathrm{Th}$ e or e m 5.1. We split the proof into three steps. The first two of them are standard and the third one applies our general Theorem 2.1. In what follows we use a random variable $X$ having the same distribution as other $X_{n}$ 's.

$\mathrm{S}$ t e p 1. Let $X_{n}^{\prime}=X_{n} I\left(\left|X_{n}\right|<n^{1 / p}\right)$. Since (c) implies (iv), the BorelCantelli lemma and $\sum_{n=1}^{\infty} \mathbf{P}\left\{|X| \geqslant n^{1 / p}\right\}<\infty$ give us $\mathbf{P}\left\{X_{n} \neq X_{n}^{\prime}\right.$ i.o. $\}=0$. For this reason in what follows we deal with $\left\{X_{n}^{\prime}\right\}$.

$\mathrm{S}$ t e p 2. Conditions (b) and (iv) assure that $n^{-1 / p} \sum_{k=1}^{n}\left|\mathbf{E} X_{k}^{\prime}\right| \rightarrow 0$, $n \rightarrow \infty$. This allows us to consider the sequence $\left\{X_{n}^{\prime}-\mathbf{E} X_{n}^{\prime}\right\}$ instead of $\left\{X_{n}^{\prime}\right\}$. It is clear that the sequence $\left\{X_{n}^{\prime}-\mathbf{E} X_{n}^{\prime}\right\}$ possesses the same Kolmogorov-Rozanov coefficient $\rho(n)$ as the original $\left\{X_{n}\right\}$ and has zero mean.

S t e p 3. Applying (5.2) to $\left\{X_{n}^{\prime}-\mathbf{E} X_{n}^{\prime}\right\}$, we obtain

$$
\mathbf{E}\left[\max _{k \leqslant n}\left|S_{k}^{\prime}\right|\right]^{2} \leqslant \text { const } 2 \theta n \exp \{\theta \varphi(n)\} \max _{j \leqslant n} \mathbf{E}\left(X_{j}^{\prime}\right)^{2},
$$

where $S_{k}^{\prime}=\sum_{j=1}^{k}\left(X_{j}^{\prime}-\mathbf{E} X_{j}^{\prime}\right)$. Set $\sigma_{n}^{2}=\max _{j \leqslant n} \mathbf{E}\left(X_{j}^{\prime}\right)^{2}$. If $F$ stays for the distribution function of $X$ then

$$
\sigma_{n}^{2}=\int_{|y|<n^{1 / p}} y^{2} d F(y)
$$

2) The notation $a_{i} \asymp b_{i}$ means that there exist two constants $0<l<L<\infty$ such that $l a_{i} \leqslant b_{i} \leqslant L b_{i}$ for all $i \geqslant 1$. 
It is clear that

$$
\begin{aligned}
& \sum_{n=1}^{\infty} \frac{\sigma_{n}^{2}}{n^{2 / p}} \exp \{\theta \varphi(n)\}=\sum_{n=1}^{\infty} \frac{\exp \{\theta \varphi(n)\}}{n^{2 / p}} \sum_{k=1}^{n} \int_{(k-1)^{1 / p} \leqslant|y|<k^{1 / p}} y^{2} d F(y) \\
& \quad=\sum_{k=1}^{\infty} \int_{(k-1)^{1 / p} \leqslant|y|<k^{1 / p}} y^{2} d F(y) \sum_{n=k}^{\infty} n^{\theta \varepsilon-2 / p} L^{\theta}(n) \\
& \leqslant \text { const } \sum_{k=1}^{\infty} k^{1+\theta \varepsilon-2 / p} L^{\theta}(k) \int_{(k-1)^{1 / p} \leqslant|y|<k^{1 / p}} y^{2} d F(y) \\
& \quad \leqslant \text { const }\left[1+\sum_{k=1}^{\infty} \int_{(k-1)^{1 / p} \leqslant|y|<k^{1 / p}}|y|^{p+p \theta \varepsilon} L^{\theta}\left(|y|^{p}\right) d F(y)\right]
\end{aligned}
$$

because $\exp \{\varphi(t)\}$ is regularly varying with the exponent $\varepsilon$. Therefore

$$
\sum_{n=1}^{\infty} \frac{\sigma_{n}^{2}}{n^{2 / p}} \exp \{\theta \varphi(n)\} \leqslant \text { const }\left[1+\mathbf{E}|X|^{p} \exp \left\{\theta \varphi\left(|X|^{p}\right)\right\}\right]<\infty
$$

Now we set $\alpha_{1}=2 \theta \exp \{\theta \varphi(1)\} \sigma_{1}^{2}$ and

$$
\alpha_{k}=2 \theta\left[k \exp \{\theta \varphi(k)\} \sigma_{k}^{2}-(k-1) \exp \{\theta \varphi(k-1)\} \sigma_{k-1}^{2}\right], \quad k>1
$$

In particular, $\alpha_{1}+\cdots+\alpha_{k}=2 \theta k \exp \{\theta \varphi(k)\} \sigma_{k}^{2}, k>1$. The series

$$
\sum k \exp \{\theta \varphi(k)\} \sigma_{k}^{2}\left[\frac{1}{k^{2 / p}}-\frac{1}{(k+1)^{2 / p}}\right]
$$

converges according to (5.4). Applying Theorem 2.1 and Remark 2.1, we prove SLLN for $\left\{X_{n}^{\prime}-\mathbf{E} X_{n}^{\prime}, n \geqslant 1\right\}$ and therefore complete the proof of Theorem 5.1.

Now we give some SLLN's for nonidentically distributed random variables. Let $q \geqslant 2$ and

$$
\varphi(n)=\sum_{i=0}^{[\ln n]} \rho\left(2^{i}\right), \quad \psi(n)=\sum_{i=0}^{[\ln n]} \rho^{2 / q}\left(2^{i}\right)
$$

Also set

$$
\sigma_{n}^{2}=\max _{j \leqslant n} \mathbf{E} X_{j}^{2}, \quad Q_{n}=\max _{j \leqslant n} \mathbf{E}\left|X_{j}\right|^{q} .
$$

$\mathrm{R}$ e $\mathrm{m}$ a $\mathrm{r} \mathrm{k}$ 5.2. We note that our approach allows one to get SLLN for sequences with $q$-th moment that are not uniformly bounded.

Theorem 5.2. Let the sequence $\left\{X_{n}, n \geqslant 1\right\}$ have KolmogorovRozanov coefficient $\rho(n)$. Assume that the constant $\theta$ is defined in (5.1). 
Assume that $\mathbf{E} X_{n}=0$ and for some $q \geqslant 2 \mathbf{E}\left|X_{n}\right|^{q}<\infty, n \geqslant 1$. Let $1 \leqslant p<2$ and

$$
\sum_{n=1}^{\infty} \frac{\sigma_{n}^{q}}{n^{1+q[1 / p-1 / 2]}} \exp \{\theta \varphi(n)\}<\infty, \quad \sum_{n=1}^{\infty} \frac{Q_{n}}{n^{q / p}} \exp \{\theta \psi(n)\}<\infty,
$$

then (5.3) holds.

Corollary 5.1. Let the sequence $\left\{X_{n}, n \geqslant 1\right\}$ have KolmogorovRozanov coefficient $\rho(n)$. Assume $\mathbf{E} X_{n}=0, \mathbf{E} X_{n}^{2}<\infty, n \geqslant 1$, and $1 \leqslant p<2$. If $\theta$ is defined in (5.2) and

$$
\sum_{n=1}^{\infty} \frac{\sigma_{n}^{2}}{n^{2 / p}} \exp \{\theta \varphi(n)\}<\infty,
$$

then (5.3) holds.

Corollary 5.2. Let the sequence $\left\{X_{n}, n \geqslant 1\right\}$ have KolmogorovRozanov coefficient $\rho(n)$. Assume $\mathbf{E} X_{n}=0, n \geqslant 1, \sup _{n \geqslant 1} \mathbf{E} X_{n}^{2}<\infty$, and $1 \leqslant p<2$. If $\theta$ is defined in (5.2) and

$$
\sum_{n=1}^{\infty} \frac{\exp \{\theta \varphi(n)\}}{n^{2 / p}}<\infty
$$

then (5.3) holds.

$\mathrm{R}$ e $\mathrm{m}$ a $\mathrm{r} \mathrm{k}$ 5.3. Note that if $\sum \rho\left(2^{i}\right)<\infty$ then (5.6) is easily satisfied. Conditions of Theorem 5.2 and Corollaries 5.1, 5.2 allows us to obtain the strong law of large numbers in the case $\sum \rho\left(2^{i}\right)=\infty$. One may use all the examples of $\rho\left(2^{i}\right)$ given in Remark 5.1 to obtain results for $\sum \rho\left(2^{i}\right)=\infty$.

P r o of of $\mathrm{T}$ h e or e $\mathrm{m}$ 5.2. First we use inequality (5.1). Then we prove that

$$
\begin{aligned}
& \sum_{n=1}^{\infty} n^{q / 2} \exp \{\theta \varphi(n)\} \sigma_{n}^{q}\left[\frac{1}{n^{q / p}}-\frac{1}{(n+1)^{q / p}}\right] \\
& \quad \leqslant \text { const } \sum_{n=1}^{\infty} \frac{\exp \{\theta \varphi(n)\}}{n^{1+q[1 / p-1 / 2]} \sigma_{n}^{q}<\infty} \\
& \sum_{n=1}^{\infty} n \exp \{\theta \psi(n)\} Q_{n}\left[\frac{1}{n^{q / p}}-\frac{1}{(n+1)^{q / p}}\right] \\
& \quad \leqslant \text { const } \sum_{n=1}^{\infty} \frac{Q_{n}}{n^{q / p}} \exp \{\theta \psi(n)\}<\infty .
\end{aligned}
$$

We refer to Theorem 2.1 and Remark 2.1 to get (5.3). The theorem is proved.

Finally, we give an estimate of the rate of growth of sums of a $\rho$-mixing sequence in terms of its natural characteristics $\sigma_{n}^{2}$ and $\rho(n)$. 
Theorem 5.3. Let $\mathbf{E} X_{n}=0$ and $\mathbf{E} X_{n}^{2}<\infty, n \geqslant 1$. Then for any $\delta>0$

$$
S_{n}=o\left(\sqrt{n \sigma_{n}^{2} \exp \{\theta \varphi(n)\}}\left[\varphi(n)+\ln \left(n \sigma_{n}^{2}\right)\right]^{1 / 2+\delta}\right) \quad \text { a.s. }
$$

In particular, if $\exp \{\varphi(t)\}=t^{\varepsilon} L(t)$, where $L$ is a slowly varying function, then

$$
S_{n}=o\left(\sqrt{n^{1+\theta \varepsilon} \sigma_{n}^{2} L^{\theta}(n)}\left[\ln \left(n \sigma_{n}^{2}\right)\right]^{1 / 2+\delta}\right) \quad \text { a.s. }
$$

P r o of. Define $\alpha_{k}$ in the same way as in Step 3 of the proof of Theorem 5.1 and let $\Lambda_{k}=\alpha_{1}+\cdots+\alpha_{k}$. Then due to an extension of an Abel theorem (see [13, Ch. IX, Lemma 15])

$$
\sum_{k=1}^{\infty} \frac{\alpha_{k}}{\Lambda_{k}\left(\ln \Lambda_{k}\right)^{1+\delta}}<\infty
$$

Now, we apply Theorem 2.1 with $b_{k}=\sqrt{\Lambda_{k}\left(\ln \Lambda_{k}\right)^{1+\delta}}$. This completes the proof of the theorem.

If $\sup _{j \geqslant 1} \sigma_{j}^{2}<\infty$ and $\sum_{i=0}^{\infty} \rho\left(2^{i}\right)<\infty$ then $S_{n}=o\left(\sqrt{n} \ln ^{1 / 2+\delta} n\right)$ a.s. for any $\delta>0$.

6. Mixingales. Introduced by McLeish [9] mixingales have been investigated by several authors. For example, Andrews [1] and Hansen [7] studied $L^{r}(r \geqslant 1)$ mixingales defined as follows. Let $\left\{\mathscr{F}_{n}, n \geqslant 1\right\}$ be a nondecreasing sequence of sub $\sigma$-fields and $\left\{X_{n}, n \geqslant 1\right\}$ be a sequence of random variables. Put $\mathbf{E}_{m} X_{i}=\mathbf{E}\left(X_{i} \mid \mathscr{F}_{m}\right)$ for $m>0$ and $\mathbf{E}_{m} X_{i}=0$ for $m \leqslant 0$. A sequence $\left(X_{n}, \mathscr{F}_{n}\right)$ is an $L^{r}$ mixingale if there exist non-negative constants $\left\{c_{i}, i \geqslant 0\right\}$ and $\left\{\psi_{m}, m \geqslant 0\right\}$ such that $\psi_{m} \downarrow 0$ and for all $i \geqslant 0$ and $m \geqslant 0$ we have

(i) $\left\|\mathbf{E}_{i-m} X_{i}\right\|_{r} \leqslant c_{i} \psi_{m}$,

(ii) $\left\|X_{i}-\mathbf{E}_{i+m} X_{i}\right\|_{r} \leqslant c_{i} \psi_{m+1}$,

where $\|\xi\|_{r}=\left(\mathbf{E}|\xi|^{r}\right)^{1 / r}$ for any random variable $\xi$. If $\left(X_{n}, \mathscr{F}_{n}\right)$ is an $L^{r}$ mixingale then it is an $L^{s}$ mixingale for $1 \leqslant s \leqslant r$. Hansen [7] got under condition $\sum_{m=1}^{\infty} \psi_{m}<\infty$ that for $r \geqslant 2$

$$
\mathbf{E}\left[\max _{k \leqslant n}\left|S_{k}\right|\right]^{r} \leqslant O(1)\left(\sum_{i=1}^{n} c_{i}^{2}\right)^{r / 2} .
$$

Using this inequality, Hansen [7] obtains the SLLN:

$$
\lim _{n \rightarrow \infty} \frac{S_{n}}{n}=0 \quad \text { a.s. }
$$

provided that $\sum c_{k}^{2} / k^{2}<\infty$ and $r \geqslant 2$. It is clear that the above SLLN is an obvious corollary of Theorem 2.1 and inequality (6.1) with $r=2$. On the other hand, Theorem 2.1 allows one to obtain more general result. 
Theorem 6.1. Let $\left\{X_{n}, n \geqslant 1\right\}$ be an $L^{2}$ mixingale such that $\psi_{m}=$ $O\left(1 /\left(\sqrt{m} \ln ^{2} m\right)\right)$ as $m \rightarrow \infty$. If $\left\{b_{n}, n \geqslant 1\right\}$ is a nondecreasing unbounded sequence and

$$
\sum_{n=1}^{\infty} \frac{c_{n}^{2}}{b_{n}^{2}}<\infty
$$

then

$$
\lim _{n \rightarrow \infty} \frac{S_{n}}{b_{n}}=0 \quad \text { a.s. }
$$

P r o of. Use Lemma 2.1 from [6] to obtain $\mathbf{E}\left[\max _{k \leqslant n}\left|S_{k}\right|\right]^{2} \leqslant$ $O(1) \sum_{k=1}^{n} c_{k}^{2}$. Now apply Theorem 2.1 .

Even better result may be obtained by using (6.1) for $r>2$.

Theorem 6.2. Let $\left\{X_{n}, n \geqslant 1\right\}$ be an $L^{r}$ mixingale, $r>2$, and $\left\{b_{n}, n \geqslant 1\right\}$ be a nondecreasing unbounded sequence. If $\sum_{m=1}^{\infty} \psi_{m}<\infty$ and

$$
\sum_{n=1}^{\infty} \frac{c_{n}^{2}}{b_{n}^{r}}\left(\sum_{k=1}^{n} c_{k}^{2}\right)^{\frac{r}{2}-1}<\infty
$$

then (6.3) holds.

$\mathrm{P}$ r $\circ$ of. This is a simple corollary of Theorem 2.1 with $\alpha_{1}=c_{1}^{r}$,

$$
\alpha_{k}=\left(\sum_{i=1}^{k} c_{i}^{2}\right)^{r / 2}-\left(\sum_{i=1}^{k-1} c_{i}^{2}\right)^{r / 2}, k>1
$$

and (6.1). We only note that

$$
\alpha_{k} \leqslant \text { const } c_{k}^{2}\left(\sum_{i=1}^{k} c_{i}^{2}\right)^{r / 2-1} .
$$

The theorem is proved.

A more precise result may be obtained in the Marcinkiewicz-Zygmund case, that is when $b_{n}=n^{1 / q}$ for $q<2$. Indeed, (6.1) implies that the condition

$$
\sum_{k=1}^{\infty} \frac{1}{k^{1+r / q}}\left(\sum_{i=1}^{k} c_{i}^{2}\right)^{r / 2}<\infty
$$

is sufficient for (6.3) in the case $b_{n}=n^{1 / q}$. Therefore the MarcinkiewiczZygmund SLLN $S_{n} / n^{1 / q} \rightarrow 0$ a.s., as $n \rightarrow \infty$, is satisfied if (6.5) holds. To compare (6.5) and (6.2) for $b_{n}=n^{1 / q}$ we set $\mu_{n}=n^{-2 / q} \sum_{k=1}^{n} c_{k}^{2}$. It is easy to see that $\sum n^{-1} \mu_{n}<\infty \Leftrightarrow \sum n^{-2 / q} c_{n}^{2}<\infty$. Therefore, if $b_{n}=n^{1 / q}$ then condition (6.2) is equivalent to $\sum n^{-1} \mu_{n}<\infty$. On the other hand,

$$
\sum_{n=1}^{\infty} \frac{1}{n^{1+r / q}}\left(\sum_{k=1}^{n} c_{k}^{2}\right)^{r / 2}=\sum_{n=1}^{\infty} \frac{1}{n} \mu_{n}^{r / 2} .
$$


Therefore, condition (6.5) is equivalent to $\sum n^{-1} \mu_{n}^{r / 2}<\infty$. Moreover, one can find a sequence $\left\{\mu_{n}\right\}$ satisfying this condition but not satisfying $\sum n^{-1} \mu_{n}<\infty$, e.g., $\mu_{n}=(\ln n)^{-1}$ and $r>2$.

7. Sequences with superadditive moment function. A sequence of random variables $\left\{X_{n}, n \geqslant 1\right\}$ is said to have the $r$-th $(r>0)$ moment function of superadditive structure if there exists a non-negative function $g(i, j)$ of two arguments such that for all $b \geqslant 0$ and $1 \leqslant k<k+l$

$$
g(b, k)+g(b+k, l) \leqslant g(b, k+l)
$$

and for some $\alpha>1$

$$
\mathbf{E}\left|S_{b, n}\right|^{r} \leqslant g^{\alpha}(b, n),
$$

where $S_{b, n}=\sum_{\nu=b+1}^{b+n} X_{\nu}$. One of possible choices for $g(i, j)$ is $g(i, j)=$ $\sum_{\nu=i+1}^{i+j} a_{\nu}^{2}$.

Under the superadditivity property Móricz [12] proved that there exists a constant $A_{r, \alpha}$ depending only on $r$ and $\alpha$ such that

$$
\mathbf{E}\left[\max _{k \leqslant n}\left|S_{k}\right|\right]^{r} \leqslant A_{r, \alpha} g_{n}^{\alpha},
$$

where $g_{n}=g(0, n)$. As $g_{n}$ increases, we may apply Theorem 2.1. For instance, put $\Lambda_{n}=g_{n}^{\alpha}$ and $\alpha_{1}=g_{1}^{\alpha}, \alpha_{k}=\Lambda_{k}-\Lambda_{k-1}, k>1$. Theorem 2.1 implies for any nondecreasing and unbounded sequence $\left\{b_{n}, n \geqslant 1\right\}$ that

$$
\lim _{n \rightarrow \infty} \frac{S_{n}}{b_{n}}=0 \text { a.s. }
$$

provided that

$$
\sum_{n=1}^{\infty} \frac{g_{n}^{\alpha}-g_{n-1}^{\alpha}}{b_{n}^{r}}<\infty
$$

If $g_{n} \uparrow \infty, \alpha>1, \delta>0$ and $b_{n}=\left[g_{n}^{\alpha} \ln g_{n}\left(\ln \ln g_{n}\right)^{1+\delta}\right]^{1 / r}$ then condition 7.2 is satisfied (see [13, Ch. IX, Lemma 15]) and Theorem 2.1 gives

$$
S_{n}=o\left(\left[g_{n}^{\alpha} \ln g_{n}\left(\ln \ln g_{n}\right)^{1+\delta}\right]^{1 / \tau}\right) \quad \text { a.s. }
$$

This is a slight generalization of Theorem 6 in [12].

Using Theorem 2.1, we also may give Marcinkiewicz-Zygmund SLLN for sequences with moment function of superadditive structure.

Theorem 7.1. Assume that a sequence of random variables $\left\{X_{n}\right.$, $n \geqslant 1\}$ has $r$-th moment function of superadditive structure with $r>0$, $\alpha>1$. Let $q>0$. If

$$
\sum_{n=1}^{\infty} \frac{g_{n}^{\alpha}}{n^{1+\frac{r}{q}}}<\infty
$$

then $\lim _{n \rightarrow \infty} S_{n} / n^{1 / q}=0$ a.s. 
P r $\circ$ of. By Remark 2.1, condition (7.3) is sufficient for (7.2) when $b_{n}=n^{1 / q}$.

8. Logarithmically weighted sums. The theorem below is a special case of a Móri [11] result. The proof given below is much shorter than that presented in [11].

Theorem 8.1. Let for some $\beta>0$ and $C>0$

$$
\left|\mathbf{E} X_{k} X_{l}\right| \leqslant C\left(\frac{l}{k}\right)^{\beta}, \quad l \leqslant k .
$$

Then

$$
\lim _{n \rightarrow \infty} \frac{1}{\ln n} \sum_{k=1}^{n} \frac{X_{k}}{k}=0 \quad \text { a.s. }
$$

P r o o f. Without loss of generality we may assume that $0<\beta<1$. Using assumption (8.1) and Lemma 8.1 below we get

$\mathbf{E}\left|\sum_{k=i}^{j} \frac{X_{k}}{k}\right|^{2} \leqslant 2 \sum_{k=i}^{j} \sum_{l=i}^{k} \frac{1}{k l}\left|\mathbf{E} X_{k} X_{l}\right| \leqslant 2 C \sum_{k=i}^{j} \sum_{l=i}^{k} \frac{1}{k^{1+\beta}} \frac{1}{l^{1-\beta}} \leqslant 2 C \frac{2}{\beta}\left(\sum_{k=i}^{j} \frac{1}{k}\right)^{\gamma}$,

where $1<\gamma<2$. Now Theorem 1 of Longnecker and Serfling [10] implies that

$$
\mathbf{E}\left[\max _{i \leqslant n}\left|\sum_{k=1}^{i} \frac{X_{k}}{k}\right|\right]^{2} \leqslant A_{2, \gamma} \frac{4 C}{\beta}\left(\sum_{k=1}^{n} \frac{1}{k}\right)^{\gamma},
$$

where $A_{2, \gamma}$ is a constant defined in [10]. Now we use $\sum_{k=1}^{n} 1 / k=O(1) \ln n$, as $n \rightarrow \infty$, to obtain

$$
\mathbf{E}\left[\max _{i \leqslant n}\left|\sum_{k=1}^{i} \frac{X_{k}}{k}\right|\right]^{2} \leqslant O(1)(\ln n)^{\gamma}
$$

Set $\alpha_{n}=(\ln (n+1))^{\gamma}-(\ln n)^{\gamma}$ and note that $\lim _{n \rightarrow \infty} \gamma n^{-1}(\ln n)^{\gamma-1} / \alpha_{n}=1$. Therefore $\sum_{n=1}^{\infty} \alpha_{n} /(\ln n)^{2}<\infty$. Thus Theorem 2.1 implies (8.2).

Lemma 8.1. Set for $i \leqslant j g(i, j)=\sum_{k=i}^{j} 1 / k$. Then for any $0<\beta<1$ and $1<\gamma<2$

$$
\sum_{k=i}^{j} \sum_{l=i}^{k} \frac{1}{k^{1+\beta}} \frac{1}{l^{1-\beta}} \leqslant \frac{2}{\beta} g^{\gamma}(i, j) .
$$

P r o of. First assume that numbers $i \leqslant j$ are such that $\sum_{k=i}^{j} 1 / k \leqslant 1$. Then

$$
\sum_{k=i}^{j} \sum_{l=i}^{k} \frac{1}{k^{1+\beta}} \frac{1}{l^{1-\beta}} \leqslant \sum_{k=i}^{j} \frac{1}{k} \sum_{l=i}^{k} \frac{1}{l} \leqslant g^{2}(i, j) \leqslant g^{\gamma}(i, j) .
$$


Otherwise, let $\sum_{k=i}^{j} 1 / k>1$. We have

$$
\sum_{l=i}^{k} \frac{1}{l^{1-\beta}} \leqslant 1+\sum_{l=2}^{k} \int_{l-1}^{l} \frac{d x}{x^{1-\beta}}=1+\left.\frac{x^{\beta}}{\beta}\right|_{x=1} ^{x=k} \leqslant 1+\frac{k^{\beta}}{\beta} \leqslant \frac{2}{\beta} k^{\beta} .
$$

Therefore

$$
\sum_{k=i}^{j} \sum_{l=i}^{k} \frac{1}{k^{1+\beta}} \frac{1}{l^{1-\beta}} \leqslant \frac{2}{\beta} g(i, j) \leqslant \frac{2}{\beta} g^{\gamma}(i, j) .
$$

Lemma is proved.

Acknowledgment. The authors are grateful to an anonymous reviewer for careful reading and suggestions improving the style of the paper.

\section{REFERENCES}

1. Andrews $D . W . K$. Laws of large numbers for dependent nonidentically distributed random variables. - Econometric Theory, 1988, v. 4, p. 458-467.

2. Brunk H. D. The strong law of large numbers. - Duke Math. J., 1948, v. 15, p. 181195.

3. Burkholder D. L. Distribution function inequalities for martingales. - Ann. Probab., 1973 , v. 1, № 1, p. 19-42.

4. Фихтенгольч $Г$. М. Курс дифференциального и интегрального исчисления. Т. 2. M.: Наука, 1969.

5. Hájek J., Rényi A. Generalization of an inequality of Kolmogorov. - Acta Math. Acad. Sci. Hungar., 1955, v. 6, № 3-4, p. 281-283.

6. Hall P., Heyde C. C. Martingale limit theory and its application. New York: Academic Press, 1980.

7. Hansen B.E. Strong laws for dependent heterogeneous processes. - Econometric Theory, 1991, v. 7, p. 213-221; 1992, v. 8, p. 421-422 (Erratum).

8. Клесов О.И., Фазекащ И. Оценки для моментов взвешенных сумм случайных величин. - Теория вероятн. и ее примен., 1997, в. 42, с. 401-402.

9. McLeish D. L. A maximal inequality and dependent strong laws. - Ann. Probab., 1975 , v. 3, № 5 , p. 829-839.

10. Longnecker M., Serfling R.J. General moment and probability inequalities for the maximum partial sum. - Acta Math. Acad. Sci. Hungar., 1977, v. 30, № 1-2, p. 129 133.

11. Móri T.F. On the strong law of large numbers for logarithmically weighted sums. Ann. Univ. Sci. Budapest, 1993, v. 36, p. 35-46.

12. Móricz $F$. Moment inequalities and the strong laws of large numbers. - $\mathrm{Z}$. Wahrscheinlichkeitstheor. verw. Geb., 1976, v. 35, p. 299-314.

13. Петров В. В. Суммы независимых случайных величин. М.: Наука, 1972, 416 с.

14. Прохоров Ю.В. Об усиленном законе больших чисел. - Изв. АН СССР, сер. матем., 1950 , т. 14 , № 6 , с. 523-536.

15. Stout W. F. Almost Sure Convergence. New York: Academic Press, 1974.

16. Qi-Man Shao. Maximal inequalities for partial sums of $\rho$-mixing sequences. - Ann. Probab., 1995, v. 23, № 2, p. 948-965. 